Rev. Elev, Méd. vét. Pays trop. 1967, 20, 2 (301-306)

\title{
Observations complémentaires faites à Madagascar sur la résistance de la tique Boophilus microplus (Canestrini) aux insecticides de la famille des hydrocarbures chlorés
}

\author{
par G. UILENBERG
}

\begin{abstract}
RÉSUMÉ
Il est confirmé que des souches de la lique Boophilus micrcplus résisiantes à I'H. C. H., possèdent une résistance croisée automatique à d'autres insecticides du même groupe, auquel nous pouvons ajouter la télodrine. Une souche a montré une résistance à la dieldrine, le toxaphène, l'endrine et la télodrine, alors que la résistance ò l'H. C. H. n'érait pas statistiquement significative, bien que celui-ci étalt le seul insecticide du groupe employé dans la ferme d'origine. Il a été possible d'augmenter en une génération de façon statistiquement significative la résistance de cette de rnıère souche à I'H. C. H., à la dieldrine et à la télodrine, en sélectionnant les descendants de femelles ayant résisté au traıtement à la dıeldrine et à l'endrine. Aucune femelle de souches non résistantes, utilisées comme témoıns, n'a pondu après traitement aux insecticides organochlorés en question, employés aux mêmes doses que pour les souches résistantes. La légère résistance d̀ l'arsenıc signalée en 1963 sur une souche résistante aux organochlorés a pu être confirmée ; d'autres souches résistantes aux organochlorés moníraient une sensibılıté normale à l'arsenic.
\end{abstract}

La résistance d'une souche de Boophilus microplus à l'hexachlorocyclohexane et à d'autres insecticides organochlorés du même groupe a été signalée à Madagascar en 1963. D'autres souches résistantes ont depuis été découvertes dans divers endroits de l'île, et les résultats des expériences sur certaınes de ces souches, ainsı que sur des souches non résistantes, sont rapportés cl-dessous, en ne considérant que les insecticides du groupe H. C. H., ef l'arsenıc.

\section{MÉTHODE}

Les expériences ont été limitées oux femelles gorgées, celles sur les larves ne s'étant pas mon- trées aussi satisfaisantes qu'en 1963, probablement parce que la résistance n'était pas aussi grande que celle de la première souche.

Lo méthode est pratiquement la même qu'en 1963. Les femelles gorgées, récoltées sur des bovins au Laborataire, sont immergées pendant 5 minutes dans le liquide à éprouver, celui-ci étant remué pendant ce temps. Ce traitement est effectué à la température ambiante du laboratoire (variant entre 20 et $25^{\circ}$ ). Les tiques sont ensuite rapidement séchées sur papier buvard, et placées dans des boîtes de Pétri, sur papier buvard, à l'étuve pour l'élevage de tiques (température de 270 , humidité relative d'environ 90 p. 100) : le lendemain, chaque 
femelle est placée (à la même étuve) dans un tube individuel, bouché au coton, numéroté. La présence ou l'absence de ponte est notée 20 jours après l'expérience, et la quantité d'œufs estimée (La production d'un petit chapelef d'œufs n'est pas complée comme ponte). Le pourcentage d'œufs éclos est estimé 40 à 45 jours après l'expérience, ainsi que le pourcentage de larves mortes. La différence avec la méthode suivie en 1963 tient dans le fait que cette fois la ponte de chaque femelle a pu être suivie individuellement; d'autre part, le contact avec l'insecticide a été moins prolongé (car les tiques restalent en contact avec le papier buvard dans la boîte de Pétrı pendant toute la durée de l'observation lors des expériences en 1963) et c'est pour cette raison qu'une comparaison avec les résultats de 1963 n'est pas tout à falt valable.

\section{ORIGINE DES SOUCHES EXPÉRIMENTÉES}

$1^{\circ}$ Souche «NANISANA 》: récoltée en 1963 sur un troupeau de bovins à Nanisana (banlieve de Tananarive) ; le propriétaire se plaignalt de ce que le détıquage à l'H. C. H., commencé environ 2 ans auparavant, n'étalt plus efficace.

20 Souche «MAHITSY》: obtenue en 1963 d'un veau de Mahıtsy (à environ $30 \mathrm{~km}$ au NordOvest de Tananarive) ; la souche était suspectée par le Service de l'Elevage de résistance à l'H. C. H. employé à la ferme en question depuis une période non précisée.

30 Souche «MAJUNGA》: envoyée en 1965 par le Service de l'Elevage de Majunga (sur la côte Nord-Ouest de Madagascar), pour détermination de l'espèce, sans mention de résıstance. Le détiquage $y$ a été falt pendant une courte période à l'H. C. H., ensuite à l'arsenic.

40 Souche «A»: récoltée en 1963 sur un bovin ò l'abattorr de Tananarive ; l'animal et les tiques étaient originaires de l'Ouest de Madagascar, régions où le détiquage n'esł guère pratqué.

50 Souche «BEZAHA »: reçue, pour détermination de l'espèce, en 1965, du Service de l'Elevage de Bezaha (région de Tuléar, SudOuest de Madagascar) ; absence totale de détiquage.

60 Souche «SAKAY»: la souche obtenue en 1962 à la Sakay (environ $150 \mathrm{~km}$ à l'Ouest de
Tananarive) ; la première souche résistante trouvée à Madagascar (Uilenberg, 1963).

70 Souche «ABATTOIR »: orıginaire en 1963 de bovıns à l'abattoir de Tananarive, bovins en provenance de l'Ouest, non ou très rarement détiqués (souche déjà -expérimentée en 1963 comme témoin pour la souche «SAKAY》).

$8^{\circ}$ Souche «B 》: originaire en 1965 d'un bovin non ou rarement détiqué de la banlieve de Tananarive.

\section{RÉSULTATS}

Les résultats des expériences sur les insecticides organochlorés sont donnés dans le Tableau 1.

Il en ressort que les souches «NANISANA» et «MAHITSY» sont résistantes à l'H. C. H., et qu'il existe une résistance croisée aux autres insectıcides expérimentés du même groupe (dieldrine, toxaphène, endrıne et télodrine), sans que ces souches aient jamais été exposées à ces produits. Les souches « $A$ » et « $B E Z A H A$ » ne sont pas résistantes à l' $H$. C. H. et elles sont sensibles à tous les insecticides expérımentés de ce groupe. La souche «MAJUNGA » est particulièrement intéressante; alors qu'un très faible pourcentage des femelles résiste à l'H. C. $H$. (isomère gamma à 0,02 p. 100), sans différence significative avec les deux souches non résistantes ( $A A$ » et «BEZAHA $)$ ), la souche est résıstante à la dieldrine, au toxaphène, à l'endrine et à la télodrine, insectıcides auxquels elle n'a pas été exposée auparavant. II semble donc que les quelques femelles ayant pondu après traitement à l'H. C. H., trahissent déjà une résistance débutante, et la résistance croisée aux autres produits du groupe est plus grande que celle à l'H. C. H. même.

Nous avons essayé d'augmenter la résistance de cette souche:

Les descendants de femelles ayant pondu normalement après traitement à la dieldrine ou à l'endrine, en choisissant uniquement des lots de larves vigoureuses et dont l'éclosion était normale, ont été mis sur des bovins au laboratoire ; les femelles gorgées qui en résultaient, ont été tratées à l'H. C. H., à la dieldrine et à la télodrine. Les résultats sont donnés dans le Tableau II. 
TABLEAEU $N^{\circ} \mathrm{I}$



$N=$ nombre de tiques.

$\mathrm{P}=$ nombre de tiques ayant pondu. Limites de confiance à 5 p.100.

$\mathrm{P}_{1}-\mathrm{P}_{2}=$ pourcentage de tiques ayant pondu.

$Q=$ quantité d'oeufs pondus, exprimée en pourcentage de ce que serait la quantitê normale pour le lot entier. (La quantité étaît estimée individuellement pour chaque tique et la mojenne indiquêe est celle du 1ot).

$E$ = pourcentage des oeuf $\mathrm{s}$ éclos (estimê pour chaque lot d'oeufs d'une femelle; le chiffre Indique la moyenne pour les femelles qui avaient pondu).

$\mathrm{ML}$ = pourcentage des larves mortes, peu après leur éclosion, 40 à 45 jours après le traitement des femelles. (Le pourcentage était estimé pour chaque lot de larves, et le chiffre indique la moyenne).

\section{TABLEAUU $\mathrm{N}^{\circ} \mathrm{II}$}

(Souche Majunga , génération 1ssue de femelles ayant résisté aux insecticídes du groupe H.C.H.)

\begin{tabular}{|c|c|c|c|c|c|c|c|c|c|c|c|}
\hline Produit & $\mathbb{N}$ & $\mathbf{P}$ & $P_{1}-P_{2}$ & & & Q & &  & \multicolumn{3}{|c|}{ MI. } \\
\hline Eau (têmoins) & 27 & 26 & $81,1-99,9 p$ & 100 & 96 & 100 & 95 & 100 & $<$ & $5 p$ & 100 \\
\hline $\begin{array}{l}\text { H.C.H. (ganma) } \\
\text { à } 0,02 \text { p.100 }\end{array}$ & 93 & 16 & $10,1-26,5$ & $1 "$ & 12 & $n$ & 4 & $"$ & $<$ & 5 & 11 \\
\hline Dieldrine à 0,05 p. 100 & 69 & 68 & $92,2-99,96$ & $"$ & 98 & $n$ & 88 & $"$ & & 15 & $"$ \\
\hline Têlodrine à $0,05 \quad "$ & 35 & 28 & $63,1-91,5$ & $"$ & 76 & $"$ & 38 & $"$ & & 45 & $"$ \\
\hline
\end{tabular}


II ressort d'une comparaison du Tableau ll au Tableau I que la deuxième génération « sélectionnée » de la souche «MAJUNGA» montre en effet une augmentation statistıquement significative de la résistance d̀ la dieldrine et à la télodrine. Le pourcentage de tiques résistantes à I'H. C. H. a également augmenté, mais la différence avec la première génération n'est pas statistiquement significative, tout au molns pour les nombres utilisés ; elle l'est par contre devenue par rapport aux souches non résistantes ( $A A$ » et «BEZAHA»).

En 1963, nous avions signalé une légère résistance à l'arsenic de la souche "SAKAY ». Les expériences faites depuis, sur cette souche et sur 7 autres, dont les résultats sont donnés dans le Tableau III, confirment cette résıstance. La souche «SAKAY» montre une différence significative

TABLEAU N ${ }^{\circ}$ III

\begin{tabular}{|c|c|c|c|c|c|c|}
\hline Souche & $\mathrm{N}$ & $\mathbf{P}$ & $P_{1}-P_{2}$ & $Q$ & $\mathrm{E}$ & ML \\
\hline \multirow{3}{*}{$\begin{array}{l}\text { Sakay } \\
\text { Abattoir }\end{array}$} & \multicolumn{6}{|c|}{$\begin{array}{l}\left.1^{\circ}\right) \text { Solution commerciale à base d"arsenite de soude et d'actde crésylique. } \\
\text { Diluée pour donner une concentration en } \mathrm{As}_{2}{ }^{0}{ }_{3} \text { de } 0,126 \text { p. } 100 \text {. }\end{array}$} \\
\hline & 28 & 15 & $34,3-72,3$ p.100 & 37 p.100 & $0 \mathrm{p} .100$ & \multirow[t]{2}{*}{ - } \\
\hline & 43 & 8 & $8,4-33,4$ & 12 & a & \\
\hline & \multicolumn{6}{|c|}{$\begin{array}{l}\left.2^{\circ}\right) \text { Solution préparée au Laboratoire, contenant de l'arsenite de soude sans acide } \\
\text { crésylique, titrếe à } 0,16 \mathrm{p} .100 \mathrm{~d}^{\prime} \mathrm{As}_{2} \mathrm{O}_{3} \text {. }\end{array}$} \\
\hline Sakay & 34 & 21 & $43,9-77,8 \mathrm{p} .100$ & $42 \mathrm{p} \cdot 100$ & $3 p+100$ & $<5$ p.100 \\
\hline Mahitsy & 68 & 22 & $21,5-44,7$ & 24 & & - \\
\hline Nanisana & 29 & 4 & $3,9-31,6$ & 9 & 0 & - \\
\hline Majunga & 26 & 3 & $2,4-30,0$ & 11 & 0 & - \\
\hline A & 52 & 11 & $11,0-34,7$ & 13 & & - \\
\hline Bezaha & 46 & 6 & $4,9-26,2$ & 12 & 0 & - \\
\hline B & 97 & 19 & $12,2-28,9$ & 17 & 0 & - \\
\hline$\underset{B}{E a u}$ (Têmoins) & 20 & 19 & $75,1-99,9$ & 85 & non mesuré & non mesurë \\
\hline
\end{tabular}

avec la souche «ABATTOIR » dans les résultats du traitement à la solution commerciale ; il y a également une différence significatıe entre la souche «SAKAY» d'une part, et les souches «NANISANA», «MAJUNGA», «A», «BE$Z A H A »$ et $« B »$ d'autre part, dans les résultats du traitement d̀ la solution d'arsenite de soude pure : la souche «MAHITSY» n'est statistıquement différente ni de la souche «SAKAY», ni des autres souches. A noter qu'il n'y a eu éclosion que de la seule souche "SAKAY» (d'un pourcentage d'alleurs très fable des œufs) après trattement d̀ l'arsenic.

\section{CONCLUSIONS ET DISCUSSION}

Nos résultats confirment que les souches devenues résistantes à l'H. C. H. par l'utilısation régulière de cet insecticide, développent automatıquement une résistance croisée aux autres insecticides du groupe, in casu la dieldrine, le toxaphène, l'endrine et la télodrine. Les résultats obtenus sur la souche «MAJUNGA» semblent montrer que cette résistance crossée peut devenir évidente avant la résistance à I'H. C. H. même; il pourrait donc être avantageux d'utiliser par exemple la dieldrine au lieu de $\mathrm{I} H$. C. $H$. pour détecter une résistance débutante à l'H. C. H.

Les résultats montrent également qu'au moindre pourcentage qui pond après traitement par un des insecticides du groupe en question (tout au moins sous la forme ef aux concentrations que nous avons employées), un début de résistance doit être soupçonné. 
II est passible, par sélection des descendants de femelles ayant résisté au traitement, d'augmenter en une génération la résistance aux insecticides du groupe H. C. H., d'où la conclusion que la sélection par le détiquage doit rapidement, une fois la résistance commencée, aboutir à toute une population de tiques résistantes dans une ferme donnée.

Les résultats obtenus soulignent une fois de plus qu'il est inutile de remplacer, en cas de

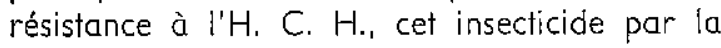
dieldrine ou le toxaphène (l'endrine et la télo- drine sont de toute façon hors de question en raison de leur toxicıté élevée).

Finalement, la résistance à l'arsenic de la souche "SAKAY » s'est montrée réelle, mais elle reste inexpliquable et ne se retrouve pas sur les autres souches résistantes aux organochlorés.

Institut d'Elevage ef de Médecine vétérinare des Pays tropicoux.

Laboratore centrol de l'élevoge (Tanonorive). Service d'Entomologie et Protozoologie.

\section{SUMMARY}

\section{« Further observations on resistance to chlorinated hydrocarbon insecticides of the tick Baophilus microplus in Madagascar 》}

It is confirmed that strains of Boophilus microplus, resistant to $B$. $H$. C., have an automatic cross-resistance to other insecticides of the same group, to which telodrin can be added. One strain showed resistance to dieldrın, toxaphene, endrin and telodrin, but resistance to B. H. C. was not statistically significant, even though this was the only insecticide of this group used on the farm concerned. It was found possible to increase in one generation, in a statistically significant way, the resistance of this strain to B. H. C., dieldrin and telodrin by selecting the descendants of females that had resisted treatment with dieldrin and endrin. Not a single female of non-resistant strains, used as contrals, layed eggs after being treated with the chlorinated hydrocarbons concerned, at the same doses as thase used on the resistant strains. The slight resistance to arsenic reported in 1963 in a sirain resistant to chlorinated hydrocarbons was confirmed; other strains resistant to chlorinated hydrocarbons showed a normal sensivity to arsenic.

\section{RES UMEN}

Observaciones complementarias sobre la resistencia a los insecticidas hidrocarburos clorados de la garrapata Boophilus microplus (Canestrini) en Madagascar

Se confirma que cepas de la garrapata Boophlus microplus resistentes al H. C. H., tienen una resistencia cruzada automática para otros insecticidas del mismo grupo, y para la telodrina. Una cepa mostró una resıstencıa a la dieldrina, el toxafeno, la endrina y la telodrina. La resistencia al H. C. H. no era significativa estadisticamente, aunque este fuera el único insecticida del grupo utilizado en la granja de que se trata. Con una generación, fué posible aumentar de manera estadısticamente signıficatıva la resistencia de la últıma cepa al H. C. H., a la dieldrina y a la telodrina, seleccionando los descendientes de las hembras que habian resistido al tratamiento con la dieldrina y la endrina. Ninguna hembra entre los cepas no resistentes, utilizadas como testigos, puso huevos después del tratamiento con los dichos insecticidas organoclorados, empleados en las mismas dosis que para las cepas resistentes. Se pudo confirmar la resistencia poco importante al arsenica notada en 1963 en una cepa resistente a los organoclorados; atras cepas resistentes a los organaclorados mostraban una sensibilidad normal para el arsenico. 


\section{BIBLIOGRAPHIE}

UILENBERG (G.). - Résistance d̀ I'hexachlorocyclohexane d'une souche de la tique Boophilus microplus (Canestrini) à Madagascar. Essais préliminaires sur sa sensibilité à quelques autres ixodicides. Rev. Elev. Méd. vét. Pays trop., 1963, $16: 137-146$. 